\title{
Research progress on permeability improvement mechanisms and technologies of coalbed deep-hole cumulative blasting
}

\author{
Deyong Guo ${ }^{1} \cdot$ Pengfei $\mathrm{Lv}^{2} \cdot$ Jiechao Zhao ${ }^{1} \cdot$ Chao Zhang ${ }^{1}$
}

Received: 24 October 2019/Revised: 14 December 2019/Accepted: 7 April 2020/Published online: 23 April 2020

(C) The Author(s) 2020

\begin{abstract}
Coalbed gas extraction is an important means of exploiting and utilizing gas resources, as well as a means of preventing coal mine disasters. In view of the low gas extraction rate from coalbeds with high gas content and low permeability, a method of improving permeability through deep-hole cumulative blasting is applied to develop initial directional fractures using a jet flow. Under the action of the blasting stress wave and detonation gas wedge, the fractures extend over a large range within the coal, thereby improving coalbed permeability. This study focuses on the criteria of cumulative blasting-induced coalbed fracturing based on a literature review of the penetration effect of cumulative blasting. On this basis, we summarize the coal fracturing zone, crack extension process, and the key technologies of charging and hole sealing for cumulative blasting. In addition, the latest research progress in the optimization of field test drilling and blasting parameters for cumulative blasting is introduced. Research findings indicate that the permeability improvement mechanism of cumulative blasting could be further enhanced, and the technology and technical equipment are in urgent need of improvement. Finally, development trends in the cumulative blasting permeability improvement technique are identified.
\end{abstract}

Keywords Cumulative blasting $\cdot$ Fracturing mechanism $\cdot$ Coalbed permeability improvement $\cdot$ Permeability improvement technology $\cdot$ Gas extraction

\section{Introduction}

Coal is the major source of energy in China, accounting for over half of primary energy production and consumption. The current energy structure and economic development stage of China suggest that coal will be the major energy source for at least the next decade (National Energy Administration 2016). As the depth of coal mining increases, the permeability of the coalbed gradually

Deyong Guo

kjkfg@cumtb.edu.cn

1 School of Emergency Management and Safety Engineering, China University of Mining and Technology, Beijing, Beijing 100083, China

2 Safety Engineering College, Beijing Institute of Petrochemical Technology, Beijing 102617, China decreases. In such cases, the risk and likelihood of coal and gas outbursts become serious. Low gas permeability restricts the improvement of coalbed gas extraction efficiency. Therefore, improving coalbed gas extraction efficiency is a key technique in preventing mine gas disasters and both exploiting and utilizing gas resources. In recent years, a great number of theoretical and experimental studies on the improvement of coalbed permeability have been carried out and a variety of techniques have been developed, such as deep-hole loosening blasting, hydraulic cutting, hydraulic fracturing, protective seam mining, and dense boreholes (Gong et al. 2006; Yao et al. 2006; Zhang et al. 2009; Liu et al. 2011). With the development and perfection of blasting theories and techniques, the permeability improvement technique of blasting fracturing has been widely applied to improve coalbed gas extraction rates, with favorable results. In particular, cumulative blasting involves directional fracture extension by means 
of the a cumulative charge structure in blasting. In 1792, mining engineer F. V. Baader discovered that the blasting energy of a cartridge with a hollow cavity structure could be accumulated. Since then, important progress in cumulative charging has been made in mining, oil and gas exploitation, and tunnel excavation. In a study by Mohaupt (1966), the cumulative effect generated by the liner on the charge cavity during cumulative charge blasting was investigated and applied to the oil and gas industry. By improving the cumulative charge structure, Bjamholt et al. (1983) developed a blasting method with a linear cumulative charge, which promoted the application of cumulative charge in directional blasting for mining, hydrocarbon production, and tunnel excavation. He et al. (2003) proposed an improved cumulative tensile blasting technique that enabled directional rock cutting using the tensile stress concentration in a certain direction in the action of bidirectional dot-bar blasting energy accumulation. The fracture surface generation problem in excavation blasting was solved by Luo et al. (2006) using a cumulative charge blasting technique. In a study by Guo et al. (2008), excessive crushing of coal during conventional blasting was overcome by means of directional coal fracturing using a unique cumulative charge structure based on the characteristics of improving coalbed permeability by blasting. Cumulative blasting has also been applied to fields such as large-area hanging control (Guo et al. 2013a), roadway driving (Yang et al. 2013; Zhu et al. 2018), and fault weakening (Gao et al. 2019), and several remarkable achievements have been reported. Moreover, Jacek et al. (2018) performed an experimental investigation of the formation and penetration efficiency of explosively formed projectile (EFP) charges with liners by applying a powder metallurgy method.

In this study, the research situation regarding fracturing mechanisms and the technologies used for coalbed deephole cumulative blasting are summarized based on progress in cumulative blasting techniques. The development trends of cumulative blasting are analyzed to provide a basis for the further optimization and improvement of coalbed deephole cumulative blasting, the prevention of gas disasters, and the more efficient exploitation and utilization of gas resources.

\section{Coalbed fracturing mechanism of deep-hole cumulative blasting}

\subsection{Studies on the penetrating effect of cumulative blasting}

In coalbed deep-hole cumulative blasting, initial directional fractures are formed in the coal by the jet flow of blasting. These fractures extend over a large range during the action of the blasting stress wave and the blasting gas wedge. With continuous research on the formation mechanism of coal fractures in the effect of blasting, many theories have been developed and numerous discoveries made, such as stress wave theory and blasting gas quasistatic compression failure theory (Yang et al. 1991), fracturing extension under the action of dynamic compression impact and stress superposition combining blasting gas (Cai et al. 2007), and dynamic and static compression failure theory ( $\mathrm{Li}$ et al. 2009). Unlike conventional blasting, the penetration effect of the jet flow generated by cumulative blasting plays an important role in directional fracturing.

Birkhoff et al. (1948) developed a metal energy liner steady collapse model according to steady incompressible fluid theory, based on which the formation mechanism of jet flow was expounded. Pugh et al. (1952) argued that the detonation wave propagated forward in the form of a spherical wave when dynamite was detonated. In the quasisteady energy liner collapse model, the time at which the wave front arrived at various particle units differed; the impact load on each particle unit and its movement velocity also varied. From the top to the bottom of the liner, the movement velocity of each particle declined. In a study by Abrahamson et al. (1963), the penetration formula of a continuous jet flow with a varying velocity was derived. The penetrating mechanism of cumulative blasting was analyzed by Shen et al. (1985) and Gong et al. (1986) based on the theory of material fracture mechanics. To handle the problems facing the metal energy liner steady collapse model, Zeng et al. (1988) proposed a quasi-steady theoretical model for jet flow formation and verified its correctness by means of numerical simulations. Based on the unique liner motion of linear-shaped charges under the Taylor bending angle, a steady-state equation of motion was derived by Seokbin (2012). In general, this can identify the liner collapse behavior based on an analytical calculation of the steady-state modified Taylor-Birkhoff theory. Salih et al. (2013) conducted an experimental investigation of the effect of the alloy composition, along with different liner geometries (conical and trumpet) and thicknesses $(4 \%, 6 \%$, and $8 \%$ charge diameter), on the penetration of aluminum shaped-charge jets against steel and sand targets.

In view of the large number of fractures and the amounts of gas that coal contains, the blasting mechanism and features of gas-containing coal and the extension rule of cumulative blasting-caused fractures were analyzed (Zhu et al. 2000) based on fracture mechanics and damage mechanics. In a study by Guo et al. (2008), the mechanical conditions of cumulative blasting for coal crack initiation, extension, and arrest and the microscopic mechanism of 
cumulative blasting directional fracturing were investigated based on fracture mechanics and explosion mechanics. The detonation process of cumulative charge is shown in Fig. 1. When the cumulative charge is detonated in the blasting hole, the liner moves to the plane that is symmetrical to it under the effect of the strong impact. The symmetry plane is thus compressed as the detonation energy accumulates. Accumulated detonation energy penetrates the coal radially along the blasting hole, thus forming initial directional macroscopic fractures in the coalbed (e.g., parts A, B, and C in Fig. 1a). Some of the energy accumulated by detonation product collision is converted into the kinetic energy of the liner for high-speed coal penetration; the rest acts upon the coal wall to form the initial fractures. These initial fractures are the joint result of the cumulative effect of detonation products and high-temperature, high-speed liner penetration. The fractures extend under the action of the detonation gas wedge. At the same time, multiple radial fractures occur due to the associated tangential tensile stress generated during the propagation of the compression stress wave caused by the detonation.

The results from a directional cumulative blasting test system indicate that the energy of directional cumulative blasting is primarily concentrated in the cumulative direction (Liu et al. 2014). The coal is penetrated to form large fractures in this direction, and these are far longer than those in non-cumulative directions. Due to the cumulative effect of directional blasting, a significant change in the stress state of the specimen is observed in the cumulative direction. The mechanical changes in the cumulative direction are greater than those in non-cumulative directions. In terms of numerical simulations, a three-dimensional model of cumulative blasting was built using an arbitrary Lagrangian-Eulerian method to investigate the formation and migration of jet flow and the process of its penetration into coal (Guo et al. 2016). Previous studies suggest that the cumulative charge structure and parameters for coal blasting are relatively simple, often referring to the relevant research results for rock blasting. Compared with rock, the internal fracturing of the coal body is more developed and its mechanical strength is lower. However, the significant differences in the internal structure and mechanical parameters of coal and rock have not been fully taken into account. Analysis of the cumulative blasting penetration effect, cumulative charge structure design, and parameter optimization were carried out without considering the specific characteristics of coal.

\subsection{Studies on the criteria and process of coal fracturing by cumulative blasting}

In terms of the criteria of coal fracturing, numerical simulations of cumulative blasting can be divided into the dynamic loading stage, static loading stage, and stabilization stage (Guo et al. 2012). Specifically, the dramatic changes in effective stress in the dynamic loading stage are the main cause of coal fracturing; stress fluctuates in a small range during the static loading stage, encouraging the coal fractures to extend; and then the stress basically remains stable in the stabilization stage, and the fractures stop extending. During the process of cumulative blasting, the coal is in a three-dimensional stress state. The stress strength (von Mises stress) at any position within it can be expressed as (Dai et al. 2001):

$\sigma_{i}=\frac{\sqrt{2}}{2}\left[\left(\sigma_{\gamma}-\sigma_{\theta}\right)^{2}+\left(\sigma_{\theta}-\sigma_{z}\right)^{2}+\left(\sigma_{z}-\sigma_{\gamma}\right)^{2}\right]^{\frac{1}{2}}$

According to von Mises' criterion of failure, a crush zone is formed by blasting when $\left(\sigma_{i}\right)_{\max } \geq S_{\mathrm{cd}}$ and a fracture development zone is formed when $\left(\sigma_{i}\right)_{\max } \geq S_{\mathrm{td}}$, where $\left(\sigma_{i}\right)_{\max }, S_{\mathrm{cd}}$, and $S_{\mathrm{td}}$ denote the peak effective stress of the coal element, and the dynamic compressive strength and dynamic tensile strength of coal, respectively. In the static loading stage, a fracture extension zone develops when $\left(\sigma_{i}\right)_{\max } \geq\left(\sigma_{i}^{\prime}\right)_{\max }$ and $\left(\sigma_{i}\right)_{\max }-\sigma^{\prime} \geq S_{\mathrm{t}}$, where $\left(\sigma_{i}^{\prime}\right)_{\max }, \sigma^{\prime}$, and $S_{\mathrm{t}}$ denote the peak effective stress of the coal element in the single action of static loading, the effective stress in the stabilization stage, and the static tensile strength of coal, respectively.

As for the process of coal fracturing by cumulative blasting, the theories and methods of the fracture

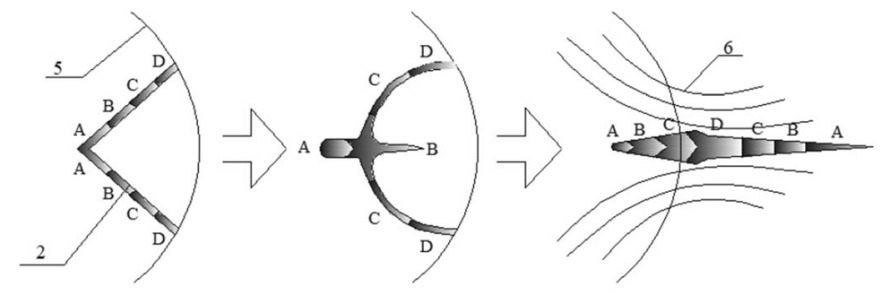

(a)

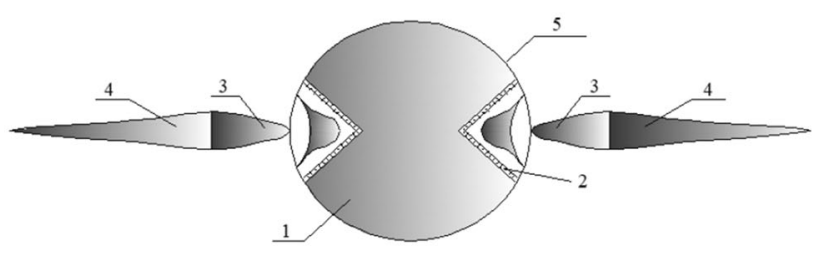

(b)

Fig. 1 Detonation process of cumulative charge (Guo et al. 2008) 1-cumulative charge; 2-liner; 3-pestle; 4-jet flow; 5-PVC tube; 6detonation product 
development directions controlled by directional cumulative blasting were investigated based on the problems of traditional coalbed permeability improvement by presplitting blasting (Mu et al. 2013). The fracture development direction and length were controlled in the joint action of the initial directional fractures generated by jet flow and the subsequent gas wedge formed by high-pressure gas. According to coal-rock blasting theory and the characteristics of the cumulative blasting process (Niu et al. 1990; Zheng et al. 2006), cumulative blasting is divided into the stages of coal penetration by jet flow, shock wave action, stress wave action, and blasting gas action. The zone of blasting influence is divided, along the radial direction of the blasting hole, into the crush zone, fracture development zone, fracture extension zone, and blasting vibration zone (Guo et al. 2012). Specifically, the crush zone is subject to the blasting shock wave that crushes the coal; the radial and circumferential cross-fractures are formed in the fracture development zone under the joint effect of the stress wave and blasting gas; radially extending fractures develop in the fracture extension zone due to the pressure of the blasting gas; and the blasting vibration zone is primarily subject to the seismic wave, for which there are few fractures. The zoning model of cumulative blasting-induced coal fractures is shown in Fig. 2.

As shown in Fig. 2, crushing coal failure occurs upon the instantaneous high pressure in the crush zone. In the cumulative direction (the direction of the blasting hole along the $x$-axis in Fig. 2), some of the accumulated energy generated by detonation products is converted into the kinetic energy of the liner for coal penetration, which makes the crush radius smaller in the $x$-direction. The crush zone is surrounded by the fracture development zone, in which radial and circumferential cross-fractures form in the non-cumulative direction (the direction of the blasting hole along the $y$-axis in Fig. 2). Due to the excessive consumption of blasting energy in the crush zone in the $y$ - direction, the fracture development zone only extends slightly in the $y$-direction. Macro-fractures are formed in the direction of the $x$-axis when the coal medium is penetrated by the liner and detonation products, and these extend in a larger range than in other directions due to the action of the stress wave and detonation gas. Blasting gas is the major force promoting fracture initiation and extension within the blasting holes in the fracture extension zone. The original stress state of coal, along with the shapes and sizes of the original fractures, changes under the action of the blasting stress wave and blasting gas pressure within the blasting hole. Thus, separate fractures intersect each other to form a fracture network. Coal fracture extension driven by coalbed gas pressure is primarily manifested through a reduction in confined pressure in the coal medium and the development of secondary fractures. Compared with the blasting gas, this plays a relatively small role.

The characteristics and range of coalbed fracture extension caused by cumulative blasting were numerically simulated based on research into the zoning model of deephole cumulative blasting-caused coalbed fractures. The results (Guo et al. 2019) indicate that the area around the blasting hole can be divided into the blast crush zone, blast fracture zone, and elastic deformation zone. According to the fracture type and the difference in the number of fractures, the blasting fracture zone is subdivided into the dense fracture zone and main fracture extension zone. The crush zone covers an oval-shaped range, i.e., smaller in the direction of liner opening than in other directions. However, the opposite is true of the ranges in the dense fracture zone and main fracture extension zone.

\subsection{Influencing factors of coal fracturing by cumulative blasting}

Coal fracturing by cumulative blasting is subject to the control of structural planes such as joints, fractures, and

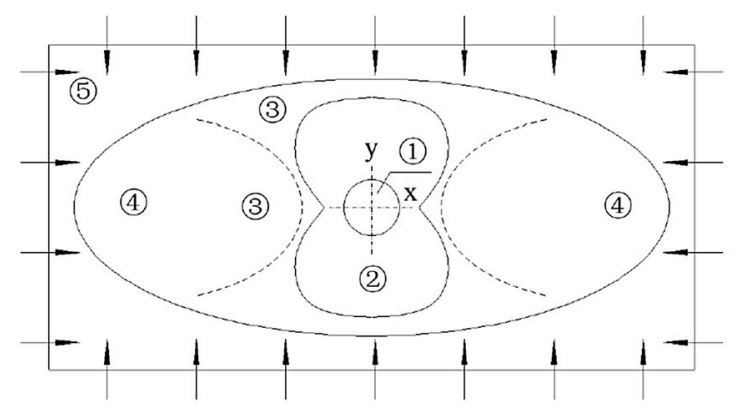

1-blasting hole; 2-crush zone; 3-fracture development zone; 4-fracture extension zone; 5-blasting vibration zone

Fig. 2 Zoning model of cumulative blasting-induced coal fractures (Guo et al. 2008) 
faults. The influence of faults on the formation and development of coal fractures caused by cumulative blasting has been studied by researchers, and the results (Guo et al. 2014) suggest that faults control the dynamic and static stress fields of the coal medium. There are significant differences in stress wave distribution and fracture extension according to the size of the fault. Significant improvements in coalbed permeability can be observed in fault-affected areas. Control holes also influence the propagation of the blasting stress wave, the extension law of the main blasting fractures, and the stress state and displacement characteristics of coal elements (Guo et al. 2018a). The stress fields around the wall of the control hole and at the tip of the main fracture were observed to change significantly under the reflection superposition of compressive stress waves at the control hole during cumulative blasting, which dominate the directional extension of main blasting fractures and the formation of circumferential fractures around the hole wall. The tangential tensile stress of the hole wall and the coal particles around it is enhanced by the displacement compensation provided by the control hole and the characteristics of the curved surface of the hole wall, which promote the development and extension of radial fractures around the hole. The control hole encourages the main blasting fractures to extend directionally, so that the radial and circumferential fractures around the hole wall cross each other and make up a large fracture network. Moreover, coalbed permeability improvement by deep-hole cumulative blasting is influenced by the charge structure (Guo et al. 2018b). With an eccentric decoupled charge, the blasting load transferred to the blasting hole wall during charged blasting is vertically asymmetric, which leads to a difference in the range of blasting fractures in the vertical direction along the blasting hole. Specifically, the fracture range at the top of the blasting hole is smaller than that at the bottom.

The above research has explored the mechanism and influence of coal fracturing by cumulative blasting from different perspectives, with some scientifically significant and practically valuable findings obtained. Due to the conditions on site and the complexity of the internal structure of coal, the process of coal fracturing by cumulative blasting depends on a variety of factors, including the geological structure, geo-stress, mining-induced stress, blasting stress, gas pressure, blasting gas, and the liquid fluid within the coal. However, researchers have failed to systematically reveal the mechanism and influence of coal fracturing caused by cumulative blasting under the coupled action of multiple factors.

\section{Permeability improvement technologies in coalbed deep-hole cumulative blasting}

\subsection{Charging technology of cumulative blasting}

In view of the difficulty of low-permeability coalbed gas extraction, Guo et al. (2009) designed a procedure containing a drill hole layout, drilling, cumulative charge structure and parameters, charging technology, hole sealing, and detonation. They then developed a whole set of technologies combining cumulative blasting coalbed fracturing theory and gas extraction techniques based on the gas geology and mining engineering conditions at Daping Coal Mine. Specifically, the charge was placed in PVC tubes to investigate the charging technology of cumulative basting. The cartridge was made from class-three permissible emulsion explosive. A directing shuttle was mounted on top of the first PVC tube and a plug placed at the end of the last PVC tube to simplify the charging. To ensure safe detonation, the cartridges were arranged to contact each other during loading; neighboring PVC tubes were firmly connected with each other. In addition, the cartridges in each PVC tube were connected with two detonators in parallel; the detonators of neighboring PVC tubes were connected in series. The explosive device was arranged in the blasting hole and the resistance was measured. The charge structure for this cumulative blasting is shown in Fig. 3.

Based on the parallel connection between the cartridges and the two detonators in each PVC tube, the detonators of neighboring PVC tubes were also connected in parallel ( $\mathrm{Lv}$ et al. 2013) to form an overall parallel connection that improves the security of the charge structure. Additionally, the charging technology in cumulative blasting field tests typically uses a circulation process involving cumulative cartridge making and charge delivery. In other words, every time a $2 \mathrm{~m}$ long cumulative cartridge is made, it is delivered to the blasting hole for a certain distance while the next cartridge is made. However, field operations suggested that too much time was spent on charge loading, and so researchers investigated continuous charging technology. Specifically, a certain number of $2 \mathrm{~m}$ long cumulative cartridges were made in advance and loaded to the blasting hole after being connecting, thereby enhancing the charging efficiency.

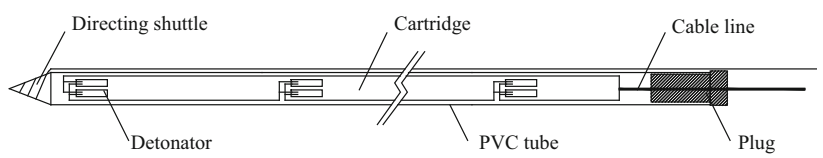

Fig. 3 Charge structure of cumulative blasting (Shang et al. 2014) 


\subsection{Cumulative blasting hole sealing technology}

Hole sealing technology is an important part of cumulative blasting. It primarily involves the selection of the hole sealing material and the determination of the hole sealing length. The hole sealing length is subject to multiple factors, such as the charge amount, hole depth, hole diameter, and gas geology of the operation area. According to practical experience obtained from cumulative blasting hole sealing experiments in Daping Coal Mine, Guo et al. (2009) identified that the quality of the hole sealing could be ensured by using yellow mud and that the hole sealing length could exceed $12 \mathrm{~m}$ considering the large amount of charge and the vulnerability of the crumbly coal wall to holes. To prevent the localized stacking of yellow mud rolls during hole sealing, adhesive yellow mud rolls with a diameter of $50 \mathrm{~mm}$ and length of $100-200 \mathrm{~mm}$ were prepared before hole sealing. They were dried and pushed into the hole, and compacted using a tamping bar on each section.

To address technical problems such as low efficiency and difficulty in ensuring the security of hole sealing when using yellow mud, the mechanical behaviors of different hole sealing materials were probed by Guo et al. (2011). They found that the blasting hole diameter and hole sealing material had a major influence on the hole sealing length. According to comprehensive analysis of the blasting security, extraction technology, and construction conditions, a reasonable sealing length following cumulative blasting was 10-12 m when using "yellow sand + yellow mud." Additionally, field tests of cumulative blasting indicated that it was difficult to blow the sand when there was a large blasting hole elevation. Thus, the hole sealing technology was improved into a three-section hole sealing structure (Fig. 4) (Shang et al. 2014). Specifically, yellow sand was filled into the inner section of the blasting hole to buffer the blasting impact. The middle section was packed by a PVC tube filled with yellow sand; a certain amount of polyurethane was attached to designated spots outside the tube using adhesive tape as a measure of tube fixation. Yellow mud rolls were then delivered and compacted into the outer section using the tamping bar, thereby ensuring the hole sealing was sufficiently resistant. Field applications suggest that hole sealing quality was enhanced by the improved hole sealing structure.

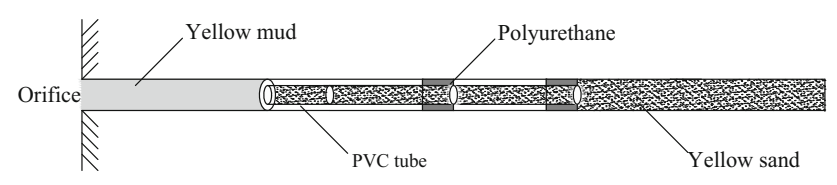

Fig. 4 Sealing structure of cumulative blasting (Shang et al. 2014)

\subsection{Cumulative blasting parameter optimization}

Coalbed deep-hole cumulative blasting can enhance coalbed permeability by promoting coal fracture development. Cumulative blasting involves many processes. The design and operation of blasthole location, drilling, charging, sealing, and detonation directly affect the coal fracturing and permeability improvement. Coal mine blasting may trigger mining disasters due to the complex and changing environment of underground blasting and a variety of artificial and uncontrollable factors during the blasting. To take advantage of cumulative blasting in coal fracturing while ensuring blasting security, the drilling parameters and blasting parameters for cumulative blasting have been investigated by many researchers.

In terms of the drilling parameters for cumulative blasting, a systematic study (Guo et al. 2013b) was carried out based on the coalbed deep-hole cumulative blasting test in Jiulishan Coal Mine, Jiaozuo Coal Group Co., Ltd. To promote coal fracture development and mitigate the crushing effect of the blasting, a radially decoupled charge structure was applied in cumulative blasting field tests. The coal fracturing effect was observed to improve when the charge diameter was $45 \mathrm{~mm}$ and the blasthole diameter was $75 \mathrm{~mm}$. A minimum spacing of $1.21 \mathrm{~m}$ between the blasthole and neighboring extraction holes was found to keep the coal wall of neighboring extraction holes from compression failure and reflected tensile failure under the propagation of the blasting stress wave. This prevents hole collapses, which could compromise the gas extraction effect. When the blasthole is close to the roof or floor of the coalbed, fractures can be formed within the rock due to the blasting effect. This brings about damage to the roof/floor stability and places the coal surface support in danger. Therefore, the spacing between the blasthole and the roof/ floor should be more than $1.5 \mathrm{~m}$. To avoid energy waste due to insufficient spacing between blasting holes and poor extraction due to excess blasting hole spacing, the rational blasting hole spacing should be set to $15-20 \mathrm{~m}$. Moreover, a comparative study on the influence of the decoupling coefficient of radial charge on the permeability effect of cumulative blasting was carried out by setting the decoupling coefficients to $1,1.33,1.67,2$, and 2.5 (Song et al. 2019). According to the results, coalbed permeability can be effectively enhanced by cumulative blasting when the decoupling coefficient is from 1.67-2.

According to research on the blasting parameters involved in coalbed fracturing and permeability improvement through cumulative blasting (Guo et al. 2013c), the action of the stress wave and blasting gas upon the coal is subject to the influence of the decoupling coefficient of the radial charge in the blasthole. If the decoupling coefficient is too large or too small, the coal fracturing will be 
negatively affected. The blasting area is determined by the axial charge length in the blasthole. As the axial charge length increases, the radial and axial pressure release ranges along the blasting hole increase and the permeability significantly improves. Not only does proper hole sealing buffer the blasting impact, it also facilitates the blasting gas to promote full extension of the coal fractures. Coal damage is subject to the influence of the number of blasting actions. With more blasting actions, the coal fractures develop more fully and become interconnected with each other in the blasting area, significantly improving coalbed permeability.

The above research mainly covers the drilling parameters and blasting parameters of cumulative blasting. Overall, relatively few factors have been considered. However, parameters such as the initial fracture, burial depth, water content, and blasting operation frequency might affect the coal fracturing and permeability improvement. At present, few of these parameters have been studied in sufficient depth.

\section{Development trends in coalbed deep-hole cumulative blasting techniques}

(1) The formation, development, and energy distribution of jet flow and its penetration effect are directly associated with the charge structure of cumulative blasting and the mechanical parameters of the liner. Optimizing the parameters of structural design and liner material selection for coalbed cumulative blasting is an important task in future studies, and the significant differences in the internal structure and mechanical parameters between coal and rock should be fully taken into account.

(2) Cumulative blasting-induced coal fracturing is subject to the joint actions of the jet flow, stress wave, high-pressure blasting gas, and mine gas. However, complex factors associated with coal and the operation environment have been simplified in existing studies on the permeability improvement mechanism of cumulative blasting. For this reason, the coupling mechanism of multiple factors (initial fracture structure, geological structure, influence of mining gas and fluids in the coal) remains to be further elucidated.

(3) According to applied research on coalbed deep-hole cumulative blasting engineering, the gas extraction rate can be significantly enhanced by cumulative blasting. Due to the complex and diversified construction environment, however, existing cumulative blasting technology and equipment have restricted practical applications. Therefore, it is still necessary to perfect the technical system of permeability improvement through coalbed deep-hole cumulative blasting and to improve the technological level and applicability of equipment. This would play a significant role in coal mining and coalbed gas development.

Acknowledgements The project was supported by the National Science Foundation of China (41430640, U1704242).

Open Access This article is licensed under a Creative Commons Attribution 4.0 International License, which permits use, sharing, adaptation, distribution and reproduction in any medium or format, as long as you give appropriate credit to the original author(s) and the source, provide a link to the Creative Commons licence, and indicate if changes were made. The images or other third party material in this article are included in the article's Creative Commons licence, unless indicated otherwise in a credit line to the material. If material is not included in the article's Creative Commons licence and your intended use is not permitted by statutory regulation or exceeds the permitted use, you will need to obtain permission directly from the copyright holder. To view a copy of this licence, visit http://creativecommons. org/licenses/by/4.0/.

\section{References}

Abrahamson GR, Goodier JN (1963) Penetration by shaped charge jets of nonuniform velocity. J Appl Phys 34(1):195-199

Birkhoff G, MacDougall DP, Pugh EM (1948) Explosives with Lined Cavities. J Appl Phys 19(6):563-582

Bjarnholt G, Holmberg R, Ouchterlong F (1983) A linear shaped charge system for contour blasting. In: Proceeding of 9th Conference on Explosives and Blasting Technique, Dallas. Society of Explosives Engineers.

Cai F, Liu ZG, Zhang CJ, Lin BQ (2007) Numerical simulation of improving permeability by deep-hole presplitting explosion in loose-soft and low permeability coal sea. J China Coal Soc 32(5):499-503

Dai J (2001) Calculation of radii of the broken and cracked areas in rock by a long charge explosion. J Liaoning Tech Univ (Nat Sci) 20(2):144-147

Gao K, Liu ZG, Liu J, Zhu FH, Qiao GD, Zhang SC (2019) Application research of directional cumulative blasting for weakening reverse faults in fully mechanized excavation face. Chin J Rock Mech Eng 38(7):1408-1419

Gong HS (1986) Fracture mechanics model of radial shaped charge cutting rock blasting. Explos Mater 1:1-3

Gong M, Liu WB, Wang DS, Wu HM, Chen TM, Qiu DC (2006) Controlled blasting technique to improve gas pre-drainage effect in a coal mine. J Univ Sci Technol Beijing 28(3):223-226

Guo DY, Pei HB, Song JC, Qin FQ, Liu XB (2008) Study on splitting mechanism of coal bed deep-hole cumulative blasting to improve permeability. J China Coal Soc 33(12):1381-1385

Guo DY, Song WJ, Li ZZ, Qin FQ, Liu XB (2009) Research on splitting technical of coal bed deep-hole cumulative blasting to improve permeability. J China Coal Soc 34(8):1086-1089

Guo DY, Yang X, Shan ZY, Lv PF (2011) Sealing technology of coal bed deep-hole cumulative blasting. J Univ Sci Technol Beijing 33(7):785-789

Guo DY, Lv PF, Pei HB, Shan ZY (2012) Numerical simulation on crack propagation of coal bed deep-hole cumulative blasting. J China Coal Soc 37(2):274-278 
Guo DY, Shang DY, Lv PF, Wang SY, Wang JM (2013a) Experimental research of deep-hole cumulative blasting in hard roof weakening. J China Coal Soc 38(7):1149-1153

Guo DY, Lv PF, Shan ZY, Xie A (2013b) Drilling parameters of deep-hole cumulative blasting to improve coal seam permeability in gas drainage. J Univ Sci Technol Beijing 35(1):16-20

Guo DY, Lv PF, Wang YG, Yang R (2013c) Blasting parameter study of deep-hole cumulative blasting to improve coal seam permeability. J Univ Sci Technol Beijing 35(12):1533-1537

Guo DY, Zhang HJ, Lv PF, Zhang GW (2014) Effect of fault on deephole cumulative blasting to improve coal bed permeability. J Univ Sci Technol Beijing 34(10):1281-1286

Guo DY, Zhao JC, Lv PF, Zhai M (2016) Dynamic effects of deephole cumulative blasting in coal seam and its application. Chin J Eng 38(12):1681-1687

Guo DY, Zhao JC, Zhang C, Zhu TG (2018a) Mechanism of control hole on coal crack initiation and propagation under deep-hole cumulative blasting in coal seam. Chin J Rock Mech Eng 37(4):919-930

Guo DY, Zhang C, Zhu TG, Pan JJ (2018b) Effect of charge structure on deep-hole cumulative blasting to improve coal seam permeability. Chin J Eng 40(12):1488-1494

Guo DY, Zhao JC, Lv PF, Zhu TG (2019) Effective fracture zone under deep-hole cumulative blasting in coal seam. Chin J Eng 41(5):582-590

He MC, Cao WF, Shan RL, Wang SL (2003) New blasting technology-bilateral cumulative tensile explosion. Chin J Rock Mech Eng 22(12):2047-2051

Jacek B, Zenon W, Piotr K, Leszek S, Bogdan Z (2018) Application of sintered liners for explosively formed projectile charges. Int $\mathrm{J}$ Impact Eng 118:91-97

Li CR, Kang LJ, Qi QX, Mao DB, Xu G (2009) Numerical simulation of deep-hole blasting and its application in mine roof weaken. J China Coal Soc 34(12):1632-1636

Liu J, Liu ZG, Gao K, Ma YK, Li ZQ, Guo LJ (2014) Experimental study and application of directional focused energy blasting in deep boreholes. Chin J Rock Mech Eng 33(12):2490-2496

Liu JC, Wang HT, Yuan ZG, Fan XG (2011) Experimental study of pre-splitting blasting enhancing pre-drainage rate of low permeability heading face. Proc Eng 26:818-823

Luo Y, Shen ZW (2006) Study on orientation fracture blasting with shaped charge in rock. Journal of University of Science and Technology Beijing 13(3):193-198

Lv PF, Dou XX, Zhu TG, Wang JM (2013) Application on permeability improved technology with deep borehole energy accumulation blasting in coal seam. Coal Sci Technol 41(12):35-38

Mohaupt H (1966) Shaped charges and warheads. Inc. Englewood Cliffs, New Jersey, pp 66-78.

Mu CM, Wang HL, Huang WY, Kuang CJ (2013) Increasing permeability mechanism using directional cumulative blasting in coal seams with high concentration of gas and low permeability. Rock Soil Mech 34(9):2496-2500

National Energy Administration (2016) The 13th Five-Year plan for coal industry development.

Niu Q (1990) Mechanism of rock blasting. Northeast Institute of Technology Press, Shenyang

Pugh EM, Eichelberger RJ, Rostoker N (1952) Theory of Jet Formation by Charges with Lined Conical Cavities. J Appl Phys 23(5):537-542

Salih S, Oguzhan A, Mehmet SY (2013) Experimental investigations on aluminum shaped charge liners. Proc Eng 58:479-486

Seokbin L (2012) Steady state equation of motion of a linear shaped charges liner. Int J Impact Eng 44:10-16

Shang DY (2014) Research on cumulative blasting technology for improving coal seam permeability and roof weakening. Dissertation, China University of Mining \& Technology, Beijing

Shen ZW (1985) Destructive effect of double-sided shaped charge jet on medium. Explos Mater 2:1-4

Song YQ, Li XS, Guo DY, Shi BK (2019) Study on the decoupled charge effect in deep-hole cumulative blasting of coal seam. Adv Civil Eng. https://doi.org/10.1155/2019/8486198

Yang RS, Zhang ZR, Yang LY, Guo YX (2013) Cumulative blasting experiment study of slotted cartridge based on hard-rock rapid driving technology. Chin J Rock Mechan Eng 32(2):317-323

Yao SW (2006) Improving method and increasing effect of gas drainage. Journal of China Coal Society 31(6):721-726

Zeng XW, Xue HL (1988) A theoretical study on linear shaped charge. Explos Shock Waves 8(2):97-105

Zhang CH, Liu ZG, Wang BS, Li LC, Zhu XH (2009) Numerical simulation and test study on mechanical properties evolution of high-pressure water injection coal seam. Chin J Rock Mech Eng 28(Supp. 2):3371-3375

Zheng PT, Yang T, Qin ZZ (2006) Theoretical modeling and analysis of the formation process of shaped charge jet. J Nat Univ Def Technol 28(3):28-32

Zhu FH, Liu ZG, Gao K, Liu J (2018) Experimental study on rock damage of roadway excavation by cumulative blasting in structural coal. Chin J Rock Mech Eng 37(9):2037-2047

Zhu XG, Luo HY, Zeng DG (2000) Analysis of coal blasting containing gas. West-China Explor Eng 66(5):97-98 